\title{
Volume-preserving exponential integrators
}

\author{
Bin Wang* $\quad$ Xinyuan $\mathrm{Wu}^{\dagger}$
}

May 31, 2018

\begin{abstract}
As is known that various dynamical systems including all Hamiltonian systems preserve volume in phase space. This qualitative geometrical property of the analytical solution should be respected in the sense of Geometric Integration. This paper analyses the volume-preserving property of exponential integrators in different vector fields. We derive a necessary and sufficient condition of volume preservation for exponential integrators, and with this condition, volumepreserving exponential integrators are analysed in detail for four kinds of vector fields. It turns out that symplectic exponential integrators can be volume preserving for a much larger class of vector fields than Hamiltonian systems. On the basis of the analysis, novel volume-preserving exponential integrators are derived for solving highly oscillatory second-order systems and extended Runge-Kutta-Nyström (ERKN) integrators of volume preservation are presented for separable partitioned systems. Moreover, the volume preservation of Runge-Kutta-Nyström (RKN) methods is also discussed. Four illustrative numerical experiments are carried out to demonstrate the notable superiority of volume-preserving exponential integrators in comparison with volume-preserving Runge-Kutta methods.
\end{abstract}

Keywords: exponential integrators, volume preservation, geometric integrators, extended RKN integrators, highly oscillatory systems

MSC (2000): 65L05, 65L06, 65L99, 34C60

\section{Introduction}

Geometric integrators (also called as structure-preserving algorithms) have been an area of great interest and active research in the recent decades. The main advantage of such methods for solving ordinary differential equations (ODEs) is that they can exactly preserve some qualitative geometrical property of the analytical solution, such as the symplecticity, energy preservation, and symmetry for long-term integration. Various geometric integrators have been designed and analysed recently and the reader is referred to [2, 3, 5, 9, 10, 12, 19, 21, 25, 27, for example on this topic. For a good theoretical foundation of geometric numerical integration for ODEs, we refer the reader to [7. 13. Surveys of structure-preserving algorithms for oscillatory differential equations are referred to 31,33 .

*School of Mathematical Sciences, Qufu Normal University, Qufu 273165, P.R.China; Mathematisches Institut, University of Tübingen, Auf der Morgenstelle 10, 72076 Tübingen, Germany. E-mail: wang@na.uni-tuebingen.de

†School of Mathematical Sciences, Qufu Normal University, Qufu 273165, P.R.China; Department of Mathematics, Nanjing University, Nanjing 210093, P.R. China. E-mail: xywu@nju.edu.cn 
It is well known that volume preservation is an important property shared by several dynamical systems. By the classical theorem due to Liouville, it is clear that all Hamiltonian systems are also of volume preservation. Preservation of volume by a numerical method has become a desirable property and many methods have been proposed and shown to be (or not to be) of volume preservation. We refer the reader to [4, 8, 14, 18, 20, 24, 35] and references therein. From the researches of this topic, it follows that all symplectic methods are of volume preservation for Hamiltonian systems. However, this result does not hold for the system beyond Hamiltonian systems. Feng and Shang have proved in [8] that no Runge-Kutta (RK) method can be of volume preservation even for linear divergence free vector fields. The authors in [4, 18] showed that no B-series method can be of volume preservation for all possible divergence-free vector fields. Thus, the design of efficient volume-preserving (VP) methods is still an open problem in the geometric numerical integration (see [22]). Recently, various VP methods have been constructed and analysed for different vector fields, such as splitting methods (see [20, 35]), RK methods (see [1]) and the methods using generating functions (see [24, 36]).

On the other hand, exponential integrators have been proposed and researched as an efficient approach to integrating ODEs/PDEs. The reader is referred to [15, 16, 17, 29] for example. However, it seems that exponential integrators with volume-preserving property for different vector fields have not been researched yet in the literature, which motives this paper.

In this paper, we study the volume preservation of exponential integrators when solving the following first-order ODEs

$$
y^{\prime}(t)=K y(t)+g(y(t)):=f(y(t)), \quad y(0)=y_{0} \in \mathbb{R}^{n},
$$

where $K$ is an $n \times n$ matrix which is assumed that $\left|e^{h K}\right| \neq-1$ for $0<h<1$, and $g: \mathbb{R}^{n} \rightarrow \mathbb{R}^{n}$ is a differentiable nonlinear function. In this paper, $|\cdot|$ denotes the determinant. The function $f$ is assumed to be divergence free such that this system is of volume preservation. It is well known that the exact solution of (10) can be presented by the variation-of-constants formula

$$
y(t)=e^{t K} y_{0}+t \int_{0}^{1} e^{(1-\tau) t K} g(y(\tau t)) d \tau .
$$

The main contributions of this paper are to derive the volume-preserving condition for exponential integrators and analyse their volume preservations for different classes of vector fields which are larger than Hamiltonian systems. Furthermore, based on the analysis, volume-preserving adapted exponential integrators are formulated for highly oscillatory second-order systems and extended Runge-Kutta-Nyström (ERKN) integrators of volume preservation are derived for separable partitioned systems. We also discuss the volume preservation of Runge-Kutta-Nyström (RKN) methods by considering them as a special class of ERKN integrators. To our knowledge, the results presented in this paper are novel that rigorously study the volume-preserving properties of exponential integrators and ERKN/RKN integrators.

We organise the remainder of this paper as follows. In Section 2, the scheme of exponential integrators is presented and some useful results of these integrators are summarised. Then a necessary and sufficient condition for exponential integrators to be of volume preservation is derived in Section 3. On the basis of this condition, we study the volume-preserving properties of exponential integrators for four kinds of vector fields in Section 4. Section 5 discusses the application to various problems including highly oscillatory second-order systems and separable partitioned systems, and shows the volume preservation of adapted exponential integrators and ERKN/RKN integrators for 
these different problems. Four illustrative numerical experiments are implemented in Section 6 and the concluding remarks are included in Section 7

\section{Exponential integrators}

In order to solve (1) effectively, one needs to approximate the integral appearing in (2) by a quadrature formula with suitable nodes $c_{1}, c_{2}, \ldots, c_{s}$. This leads to the following exponential integrators proposed in [16, which have been successfully used for solving different kinds of ODEs/PDEs.

Definition 2.1 (See [16]) An s-stage exponential integrator for numerical integration of (10) is defined by

$$
\left\{\begin{array}{l}
k_{i}=e^{c_{i} h K} y_{n}+h \sum_{j=1}^{s} \bar{a}_{i j}(h K) g\left(k_{j}\right), \quad i=1,2, \ldots, s, \\
y_{n+1}=e^{h K} y_{n}+h \sum_{i=1}^{s} \bar{b}_{i}(h K) g\left(k_{i}\right),
\end{array}\right.
$$

where $h$ is a stepsize, $c_{i}$ are real constants for $i=1, \cdots, s, \bar{b}_{i}(h K)$ and $\bar{a}_{i j}(h K)$ are matrix-valued functions of $h K$ for $i, j=1, \ldots, s$.

The coefficients of the integrator can be displayed in a Butcher tableau (omit $(h K)$ for brevity):

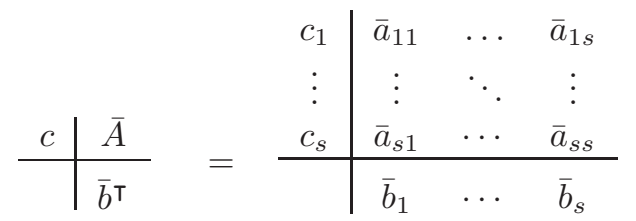

It is noted that when $K=\mathbf{0}$, this integrator reduces to a classical $s$-stage RK method represented by the Butcher tableau

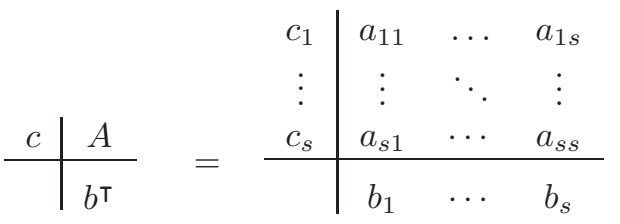

In this paper, a kind of special and important exponential integrators will be considered and analysed, which was proposed in 23 .

Definition 2.2 (See [23]) Define a kind of s-stage exponential integrators by

$$
\bar{a}_{i j}(h K)=a_{i j} e^{\left(c_{i}-c_{j}\right) h K}, \quad \bar{b}_{i}(h K)=b_{i} e^{\left(1-c_{i}\right) h K}, \quad i, j=1, \ldots, s,
$$

where

$$
c=\left(c_{1}, \ldots, c_{s}\right)^{\top}, \quad b=\left(b_{1}, \ldots, b_{s}\right)^{\top}, \quad A=\left(a_{i j}\right)_{s \times s}
$$

are the coefficients of an s-stage $R K$ method.

With regard to this kind of exponential integrators, two useful properties are shown in [23] and we summarise them as follows. 
Theorem 2.3 (See [23]) If an RK method with the coefficients (5D) is of order $p$, then the exponential integrator given by (4) is also of order $p$.

Theorem 2.4 (See [23]) The exponential integrator defined by (44) is symplectic if the corresponding RK method (5) is symplectic.

In this paper, we supplement an additional requirement to $b$ of (5) and define the following special symplectic exponential integrators (SSEI).

Definition 2.5 An s-stage exponential integrator (4) is called as special symplectic exponential integrator (SSEI) if the $R K$ method (5) is symplectic and $b_{j} \neq 0$ for all $j=1, \ldots, s$.

Remark 2.6 We note that a kind of special symplectic RK (SSRK) methods was considered in [1] and our SSEI integrators reduce to the SSRK methods when $K=\mathbf{0}$.

\section{VP condition of exponential integrators}

For each stepsize $h$, denote the $s$-stage exponential integrator (3) by a map $\varphi_{h}: \mathbb{R}^{n} \rightarrow \mathbb{R}^{n}$, which is

$$
\left\{\begin{array}{l}
\varphi_{h}(y)=e^{h K} y+h \sum_{i=1}^{s} \bar{b}_{i}(h K) g\left(k_{i}(y)\right), \\
k_{i}(y)=e^{c_{i} h K} y+h \sum_{j=1}^{s} \bar{a}_{i j}(h K) g\left(k_{j}(y)\right), \quad i=1,2, \ldots, s .
\end{array}\right.
$$

It is noted that $k_{i}(y)$ for $i=1,2, \ldots$ are identical to $\varphi_{c_{i} h}(y)$ for $i=1,2, \ldots$ in (6).

We firstly derive the Jacobian matrix and its determinant for the general exponential integrator (6).

Lemma 3.1 The Jacobian matrix of the exponential integrator (6) can be expressed as

$$
\varphi_{h}^{\prime}(y)=e^{h K}+h \bar{b}^{\top} F\left(I_{s} \otimes I-h \bar{A} F\right)^{-1} e^{c h K},
$$

where $F=\operatorname{diag}\left(g^{\prime}\left(k_{1}\right), \ldots, g^{\prime}\left(k_{s}\right)\right), I_{s}$ and $I$ are the $s \times s$ and $n \times n$ identity matrices, respectively, and $e^{c h K}=\left(e^{c_{1} h K}, \ldots, e^{c_{s} h K}\right)^{\top}$. Its determinant reads

$$
\left|\varphi_{h}^{\prime}(y)\right|=\frac{\left|e^{h K}\right|\left|I_{s} \otimes I-h\left(\bar{A}-e^{(c-1) h K} \bar{b}^{\top}\right) F\right|}{\left|I_{s} \otimes I-h \bar{A} F\right|},
$$

where 1 is an $s \times 1$ vector of units and $e^{(c-1) h K}=\left(e^{\left(c_{1}-1\right) h K}, \ldots, e^{\left(c_{s}-1\right) h K}\right)^{\top}$. Here we make use of the Kronecker product $\otimes$ throughout this paper.

Proof According to the first formula of (6), we obtain

$$
\varphi_{h}^{\prime}(y)=e^{h K}+h \sum_{i=1}^{s} \bar{b}_{i} g^{\prime}\left(k_{i}(y)\right) k_{i}^{\prime}(y)=e^{h K}+h \bar{b}^{\top} F\left(k_{1}^{\prime}, \ldots, k_{s}^{\prime}\right)^{\top}
$$


Likewise, it follows from $k_{i}(y)$ in (6) that

$$
\left(\begin{array}{cccc}
I-h \bar{a}_{11} g^{\prime}\left(k_{1}\right) & -h \bar{a}_{12} g^{\prime}\left(k_{2}\right) & \cdots & -h \bar{a}_{1 s} g^{\prime}\left(k_{s}\right) \\
-h \bar{a}_{21} g^{\prime}\left(k_{1}\right) & I-h \bar{a}_{22} g^{\prime}\left(k_{2}\right) & \cdots & -h \bar{a}_{2 s} g^{\prime}\left(k_{s}\right) \\
\vdots & \vdots & \vdots & \vdots \\
-h \bar{a}_{s 1} g^{\prime}\left(k_{1}\right) & -h \bar{a}_{s 2} g^{\prime}\left(k_{2}\right) & \cdots & I-h \bar{a}_{s s} g^{\prime}\left(k_{s}\right)
\end{array}\right)\left(\begin{array}{c}
k_{1}^{\prime} \\
k_{2}^{\prime} \\
\vdots \\
k_{s}^{\prime}
\end{array}\right)=e^{c h K},
$$

which can be rewritten as

$$
\left(I_{s} \otimes I-h \bar{A} F\right)\left(k_{1}^{\prime}, \ldots, k_{s}^{\prime}\right)^{\top}=e^{c h K} .
$$

Substituting (9) into (8) yields the first statement of this lemma.

For the second statement, we will use the following block determinant identity (see [1, 13]):

$$
|U|\left|X-W U^{-1} V\right|=\left|\begin{array}{cc}
U & V \\
W & X
\end{array}\right|=|X|\left|U-V X^{-1} W\right|,
$$

which is yielded from Gaussian elimination. By letting

$$
X=e^{h K}, W=-h \bar{b}^{\top} F, U=I_{s} \otimes I-h \bar{A} F, V=e^{c h K},
$$

it is obtained that

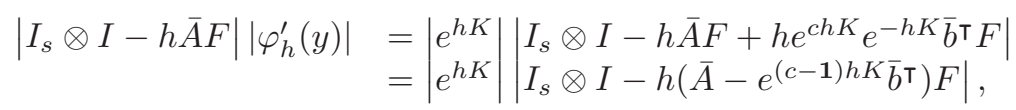

which leads to the second result (7).

For the SSEI methods of Definition 2.5, a necessary and sufficient condition for these integrators to be of volume preservation is shown by the following lemma.

Lemma 3.2 An s-stage SSEI method defined in Definition 2.5 is of volume preservation if and only if the following VP condition is satisfied

$$
\left|I_{s} \otimes I-h(A \otimes I . * E(h K)) F\right|=\left|e^{h K}\right|\left|I_{s} \otimes I+h\left(A^{\boldsymbol{\top}} \otimes I . * E(h K)\right) F\right|,
$$

where $E(h K)$ is a block matrix defined by

$$
E(h K)=\left(E_{i, j}(h K)\right)_{s \times s}=\left(\begin{array}{cccc}
I & e^{\left(c_{1}-c_{2}\right) h K} & \cdots & e^{\left(c_{1}-c_{s}\right) h K} \\
e^{\left(c_{2}-c_{1}\right) h K} & I & \cdots & e^{\left(c_{2}-c_{s}\right) h K} \\
\vdots & \vdots & \ddots & \vdots \\
e^{\left(c_{s}-c_{1}\right) h K} & e^{\left(c_{s}-c_{2}\right) h K} & \cdots & I
\end{array}\right),
$$

and .* denotes the element-wise multiplication of two matrices.

Proof In terms of the choice (44) of the coefficients, it is computed that

$$
\begin{aligned}
& \bar{A}-e^{(c-\mathbf{1}) h K} \bar{b}^{\top} \\
= & (A \otimes I) \cdot * E(h K)-\left(e^{\left(c_{1}-1\right) h K}, \ldots, e^{\left(c_{s}-1\right) h K}\right)^{\top}\left(b_{1} e^{\left(1-c_{1}\right) h K}, \ldots, b_{s} e^{\left(1-c_{s}\right) h K}\right) \\
= & (A \otimes I) \cdot * E(h K)-\left(\mathbf{1} b^{\top} \otimes I\right) . * E(h K) \\
= & \left(A-\mathbf{1} b^{\top}\right) \otimes I . * E(h K) .
\end{aligned}
$$


Thus, we obtain

$$
\left|\varphi_{h}^{\prime}(y)\right|=\frac{\left|e^{h K}\right|\left|I_{s} \otimes I-h\left(A-\mathbf{1} b^{\top}\right) \otimes I . * E(h K) F\right|}{\left|I_{s} \otimes I-h \bar{A} F\right|} .
$$

Moreover, with careful calculations, it can be verified that for $B=\operatorname{diag}\left(b_{1}, \ldots, b_{s}\right)$, the following result holds

$$
\begin{aligned}
& \left|I_{s} \otimes I-h\left(A-\mathbf{1} b^{\top}\right) \otimes I . * E(h K) F\right| \\
& =\left|I_{s} \otimes I-h(B \otimes I)\left(A-\mathbf{1} b^{\top}\right) \otimes I . * E(h K) F\left(B^{-1} \otimes I\right)\right| \\
& =\mid I_{s} \otimes I-h(B \otimes I)\left(A-\mathbf{1} b^{\top}\right) \otimes I . * E(h K)\left(B^{-1} \otimes I\right) F \\
& =\left|I_{s} \otimes I-h B\left(A-\mathbf{1} b^{\top}\right) B^{-1} \otimes I . * E(h K) F\right| .
\end{aligned}
$$

Since the RK method is symplectic, one has that $B A+A^{\top} B-b b^{\top}=0$ (see [13]), which leads to $B\left(A-\mathbf{1} b^{\top}\right) B^{-1}=-A^{\top}$. Therefore, the result (13) can be simplified as

$$
\left|I_{s} \otimes I-h\left(A-\mathbf{1} b^{\top}\right) \otimes I . * E(h K) F\right|=\left|I_{s} \otimes I+h\left(A^{\top} \otimes I . * E(h K)\right) F\right| .
$$

The proof is complete by considering (12).

Remark 3.3 It is noted that when $K=\mathbf{0}$, this VP condition (10) reduces to the condition of $R K$ methods presented in [1]. Consequently, the condition (10) can be regarded as a generalisation of that of RK methods.

\section{VP results for different vector fields}

In this section, we study the volume-preserving properties of exponential integrators for four kinds of vector fields, which are defined as follows.

Definition 4.1 (See [1]) Define the following four classes of vector fields on Euclidean space using vector fields $f(y)$

$$
\begin{aligned}
& \mathcal{H}=\left\{f \mid \text { there exists } P \text { such that for all } y, P f^{\prime}(y) P^{-1}=-f^{\prime}(y)^{\top}\right\} \text {, } \\
& \mathcal{S}=\quad\left\{f \mid \text { there exists } P \text { such that for all } y, P f^{\prime}(y) P^{-1}=-f^{\prime}(y)\right\} \text {, } \\
& \mathcal{F}^{(\infty)}=\left\{f\left(y_{1}, y_{2}\right)=\left(u\left(y_{1}\right), v\left(y_{1}, y_{2}\right)\right)^{\top} \text { where } u \in \mathcal{H} \cup \mathcal{F}^{(\infty)} \mid \text { there exists } P\right. \\
& \text { such that for all } \left.y_{1}, y_{2}, P \partial_{y_{2}} v\left(y_{1}, y_{2}\right) P^{-1}=-\partial_{y_{2}} v\left(y_{1}, y_{2}\right)^{\top}\right\} \text {, } \\
& \mathcal{F}^{(2)}=\left\{f\left(y_{1}, y_{2}\right)=\left(u\left(y_{1}\right), v\left(y_{1}, y_{2}\right)\right)^{\top} \text { where } u \in \mathcal{H} \cup \mathcal{S} \cup \mathcal{F}^{(2)} \mid \text { there exists } P\right. \\
& \text { such that for all } y_{1}, y_{2} \text {, either } P \partial_{y_{2}} v\left(y_{1}, y_{2}\right) P^{-1}=-\partial_{y_{2}} v\left(y_{1}, y_{2}\right)^{\top} \text {, } \\
& \text { or } \left.P \partial_{y_{2}} v\left(y_{1}, y_{2}\right) P^{-1}=-\partial_{y_{2}} v\left(y_{1}, y_{2}\right)\right\} \text {. }
\end{aligned}
$$

Remark 4.2 It has been proved in [1] that all these sets are equal to divergence free vector fields. The relationships of these vector fields are also given in [1] by $\mathcal{H} \subset \mathcal{F}^{(\infty)} \subset \mathcal{F}^{(2)}$ and $\mathcal{S} \subset \mathcal{F}^{(\infty)} \subset$ $\mathcal{F}^{(2)}$. According to Lemma 3.2 of [1], we know that the set $\mathcal{H}$ contains all Hamiltonian systems. Denote by $H$ the set of Hamiltonian systems. See Figure 1 for the venn diagram illusting the relationships. This figure clearly shows that the sets $\mathcal{H}, \mathcal{F}^{(\infty)}$ and $\mathcal{F}^{(2)}$ are larger classes of vector fields than Hamiltonian systems. 


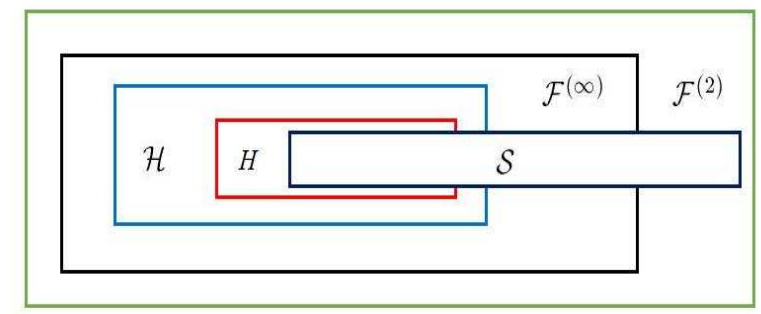

Figure 1: Venn diagram illusting the relationships.

\subsection{Vector field $\mathcal{H}$}

Theorem 4.3 All SSEI methods for solving (1) are of volume preservation for vector fields $f$ and $g$ in $\mathcal{H}$ with the same $P$.

Proof Let $P$ be such that for all $y, P f^{\prime}(y) P^{-1}=-f^{\prime}(y)^{\top}$ and $P g^{\prime}(y) P^{-1}=-g^{\prime}(y)^{\top}$. According to this condition and the expression $f(y)=K y+g(y)$, one has that $P K P^{-1}=-K^{\top}$. Thus it is easily obtained that $P e^{h K} P^{-1}=e^{-h K^{\top}}$. In the light of this result, one gets

$$
\left|P e^{h K} P^{-1}\right|=\left|e^{h K}\right|=\left|e^{-h K^{\top}}\right|=\left|e^{-h K}\right|=\left|\left(e^{h K}\right)^{-1}\right|=\frac{1}{\mid e^{h K}},
$$

which yields $\left|e^{h K}\right|=1$ (it is assumed that $\left|e^{h K}\right| \neq-1$ in the introduction of this paper). We then compute the left-hand side of (10) as follows

$$
\begin{aligned}
& \left|I_{s} \otimes I-h(A \otimes I . * E(h K)) F\right| \\
= & \left|\left(I_{s} \otimes P\right)\left(I_{s} \otimes P^{-1}\right)-h\left(I_{s} \otimes P\right)(A \otimes I . * E(h K))\left(I_{s} \otimes P^{-1}\right)\left(I_{s} \otimes P\right) F\left(I_{s} \otimes P^{-1}\right)\right| \\
= & \left|I_{s} \otimes I+h\left(I_{s} \otimes P\right)(A \otimes I . * E(h K))\left(I_{s} \otimes P^{-1}\right) F^{\top}\right| \\
= & \left|I_{s} \otimes I+h\left(A \otimes I . * E\left(-h K^{\top}\right)\right) F^{\top}\right| \\
= & \left|I_{s} \otimes I+h F\left(A^{\top} \otimes I . * E\left(-h K^{\top}\right)^{\top}\right)\right| \text { (transpose) } \\
= & \left|I_{s} \otimes I+h\left(A^{\top} \otimes I . * E\left(-h K^{\top}\right)^{\top}\right) F\right| \text { (Sylvester's law). }
\end{aligned}
$$

It follows from the definition (11) that

$$
E\left(-h K^{\top}\right)^{\top}=\left(E_{i, j}\left(-h K^{\top}\right)\right)_{s \times s}^{\top}=\left(E_{j, i}(-h K)\right)_{s \times s}=\left(E_{i, j}(h K)\right)_{s \times s}=E(h K) .
$$

Therefore, one arrives at

$$
\left|I_{s} \otimes I-h(A \otimes I . * E(h K)) F\right|=\left|I_{s} \otimes I+h\left(A^{\top} \otimes I . * E(h K)\right) F\right|,
$$

which shows that all SSEI methods are of volume preservation for vector fields in $\mathcal{H}$ by considering Lemma 3.2 .

\subsection{Vector field $\mathcal{S}$}

Theorem 4.4 All one-stage SSEI methods and all two-stage SSEI methods with $c_{1}=c_{2}$ (and any composition of such methods) are of volume preservation for vector fields $f$ and $g$ in $\mathcal{S}$ with the same $P$. 
Proof Let $P$ be such that for all $y, P f^{\prime}(y) P^{-1}=-f^{\prime}(y)$ and $P g^{\prime}(y) P^{-1}=-g^{\prime}(y)$. Similarly to the proof of last theorem, we obtain that $P K P^{-1}=-K$. Thus it is true that $P e^{h K} P^{-1}=e^{-h K}$ and $\left|e^{h K}\right|=1$.

For the one-stage SSEI, according to Lemma 3.2, it is of volume preservation if and only if

$$
\left|I-h a_{11} g^{\prime}\left(k_{1}\right)\right|=\left|I+h a_{11} g^{\prime}\left(k_{1}\right)\right|,
$$

which can be verified by considering

$$
\left|I-h a_{11} g^{\prime}\left(k_{1}\right)\right|=\left|P P^{-1}-h a_{11} P g^{\prime}\left(k_{1}\right) P^{-1}\right|=\left|I+h a_{11} g^{\prime}\left(k_{1}\right)\right| .
$$

For a two-stage SSEI method, according to Lemma 3.2 again, this two-stage SSEI method is of volume preservation if and only if

$$
=\left|\begin{array}{cc}
I-h a_{11} g^{\prime}\left(k_{1}\right) & -h a_{12} e^{\left(c_{1}-c_{2}\right) h K} g^{\prime}\left(k_{2}\right) \\
-h a_{21} e^{\left(c_{2}-c_{1}\right) h K} g^{\prime}\left(k_{1}\right) & I-h a_{22} g^{\prime}\left(k_{2}\right) \\
I+h a_{11} g^{\prime}\left(k_{1}\right) & h a_{21} e^{\left(c_{1}-c_{2}\right) h K} g^{\prime}\left(k_{2}\right) \\
h a_{12} e^{\left(c_{2}-c_{1}\right) h K} g^{\prime}\left(k_{1}\right) & I+h a_{22} g^{\prime}\left(k_{2}\right)
\end{array}\right|,
$$

which gives the condition

$$
\begin{aligned}
& \mid I-h a_{11} g^{\prime}\left(k_{1}\right)-h a_{22} g^{\prime}\left(k_{2}\right)+h^{2} a_{11} a_{22} g^{\prime}\left(k_{1}\right) g^{\prime}\left(k_{2}\right) \\
& -h^{2} a_{12} a_{21} e^{\left(c_{1}-c_{2}\right) h K} g^{\prime}\left(k_{2}\right) e^{\left(c_{2}-c_{1}\right) h K} g^{\prime}\left(k_{1}\right) \mid \\
= & \mid I+h a_{11} g^{\prime}\left(k_{1}\right)+h a_{22} g^{\prime}\left(k_{2}\right)+h^{2} a_{11} a_{22} g^{\prime}\left(k_{1}\right) g^{\prime}\left(k_{2}\right) \\
& -h^{2} a_{12} a_{21} e^{\left(c_{1}-c_{2}\right) h K} g^{\prime}\left(k_{2}\right) e^{\left(c_{2}-c_{1}\right) h K} g^{\prime}\left(k_{1}\right) \mid .
\end{aligned}
$$

It also can be verified that

$$
\begin{aligned}
& \text { the left hand side of }(15) \\
= & \mid P P^{-1}-h a_{11} P g^{\prime}\left(k_{1}\right) P^{-1}-h a_{22} P g^{\prime}\left(k_{2}\right) P^{-1}+h^{2} a_{11} a_{22} P g^{\prime}\left(k_{1}\right) g^{\prime}\left(k_{2}\right) P^{-1} \\
& -h^{2} a_{12} a_{21} P e^{\left(c_{1}-c_{2}\right) h K} g^{\prime}\left(k_{2}\right) e^{\left(c_{2}-c_{1}\right) h K} g^{\prime}\left(k_{1}\right) P^{-1} \mid \\
= & \mid I+h a_{11} g^{\prime}\left(k_{1}\right)+h a_{22} g^{\prime}\left(k_{2}\right)+h^{2} a_{11} a_{22} P g^{\prime}\left(k_{1}\right) P^{-1} P g^{\prime}\left(k_{2}\right) P^{-1} \\
& -h^{2} a_{12} a_{21} P e^{\left(c_{1}-c_{2}\right) h K} P^{-1} P g^{\prime}\left(k_{2}\right) P^{-1} P e^{\left(c_{2}-c_{1}\right) h K} P^{-1} P g^{\prime}\left(k_{1}\right) P^{-1} \mid \\
= & \mid I+h a_{11} g^{\prime}\left(k_{1}\right)+h a_{22} g^{\prime}\left(k_{2}\right)+h^{2} a_{11} a_{22} g^{\prime}\left(k_{1}\right) g^{\prime}\left(k_{2}\right) \\
& -h^{2} a_{12} a_{21} e^{\left(c_{2}-c_{1}\right) h K} g^{\prime}\left(k_{2}\right) e^{\left(c_{1}-c_{2}\right) h K} g^{\prime}\left(k_{1}\right) \mid .
\end{aligned}
$$

Under the assumption that $c_{1}=c_{2}$, this result becomes

$$
\begin{aligned}
& \mid I+h a_{11} g^{\prime}\left(k_{1}\right)+h a_{22} g^{\prime}\left(k_{2}\right)+h^{2} a_{11} a_{22} g^{\prime}\left(k_{1}\right) g^{\prime}\left(k_{2}\right) \\
& \quad-h^{2} a_{12} a_{21} e^{\left(c_{1}-c_{2}\right) h K} g^{\prime}\left(k_{2}\right) e^{\left(c_{2}-c_{1}\right) h K} g^{\prime}\left(k_{1}\right) \mid .
\end{aligned}
$$

Thus (15) is obtained immediately and then all two-stage SSEI methods with $c_{1}=c_{2}$ are of volume preservation.

Remark 4.5 It is noted that for the vector field $\mathcal{S}$ and two-stage SSEI methods, the condition

$$
c_{1}=c_{2}
$$

is supplemented in order to make the following condition be true

$$
e^{\left(c_{2}-c_{1}\right) h K} g^{\prime}\left(k_{2}\right) e^{\left(c_{1}-c_{2}\right) h K} g^{\prime}\left(k_{1}\right)=e^{\left(c_{1}-c_{2}\right) h K} g^{\prime}\left(k_{2}\right) e^{\left(c_{2}-c_{1}\right) h K} g^{\prime}\left(k_{1}\right) .
$$

We remark that condition (16) is not necessary for some special matrix $K$ such as $K=0$ or some special function $g$ such as scalar functions. The same situation happens in the analysis of Subsection 4.4 


\subsection{Vector field $\mathcal{F}^{(\infty)}$}

For the vector field $\mathcal{F}^{(\infty)}$, if the function $f(y):=K y+g(y)$ has the pattern $\left(u\left(y_{1}\right), v\left(y_{1}, y_{2}\right)\right)^{\top}$, this means that $K$ and $g$ can be expressed in blocks as

$$
K=\left(\begin{array}{cc}
K_{11} & 0 \\
0 & K_{22}
\end{array}\right), \quad g(y)=\left(\begin{array}{c}
g_{1}\left(y_{1}\right) \\
g_{2}\left(y_{1}, y_{2}\right)
\end{array}\right) .
$$

Then the following relation is true

$$
u\left(y_{1}\right)=K_{11} y_{1}+g_{1}\left(y_{1}\right), \quad v\left(y_{1}, y_{2}\right)=K_{22} y_{2}+g_{2}\left(y_{1}, y_{2}\right) .
$$

Theorem 4.6 Consider an s-stage SSEI method for solving $y_{1}^{\prime}=u\left(y_{1}\right)$ that is of volume preservation for the vector field $u\left(y_{1}\right): \mathbb{R}^{m} \rightarrow \mathbb{R}^{m}$. Let $v\left(y_{1}, y_{2}\right): \mathbb{R}^{m+n} \rightarrow \mathbb{R}^{m+n}$ and assume that there exists an invertible matrix $P$ such that for all $y_{1}, y_{2}$,

$$
P \partial_{y_{2}} v\left(y_{1}, y_{2}\right) P^{-1}=-\partial_{y_{2}} v\left(y_{1}, y_{2}\right)^{\top}, \quad P \partial_{y_{2}} g_{2}\left(y_{1}, y_{2}\right) P^{-1}=-\partial_{y_{2}} g_{2}\left(y_{1}, y_{2}\right)^{\top} .
$$

Then the SSEI method is of volume preservation for vector fields $f\left(y_{1}, y_{2}\right)=\left(u\left(y_{1}\right), v\left(y_{1}, y_{2}\right)\right)^{\top}$ in $\mathcal{F}^{(\infty)}$.

Proof From the property of $v$, we have $P K_{22} P^{-1}=-K_{22}^{\top}$ and $\left|e^{h K_{22}}\right|=1$. Thus $\left|e^{h K}\right|=$ $\left|e^{h K_{11}}\right|\left|e^{h K_{22}}\right|=\left|e^{h K_{11}}\right|$. The Jacobian matrix of $g(y)$ is block triangular as follows

$$
g^{\prime}\left(y_{1}, y_{2}\right)=\left(\begin{array}{cc}
\partial_{y_{1}} g_{1}\left(y_{1}\right) & 0 \\
* & \partial_{y_{2}} g_{2}\left(y_{1}, y_{2}\right)
\end{array}\right) .
$$

In what follows, we prove the condition (10). Using the block transformation, we can bring the left-hand side of (10) to the block form

$$
\left|I_{s} \otimes I-h(A \otimes I . * E(h K)) F\right|=\left(\begin{array}{cc}
\Phi_{1} & 0 \\
* & \Phi_{2}
\end{array}\right),
$$

where

$$
\Phi_{1}=\left(\begin{array}{ccc}
I-h \bar{a}_{11}\left(h K_{11}\right) \partial_{y_{1}} g_{1}\left(k_{1}\right) & \cdots & -h \bar{a}_{1 s}\left(h K_{11}\right) \partial_{y_{1}} g_{1}\left(k_{s}\right) \\
\vdots & \ddots & \vdots \\
-h \bar{a}_{s 1}\left(h K_{11}\right) \partial_{y_{1}} g_{1}\left(k_{1}\right) & \cdots & I-h \bar{a}_{s s}\left(h K_{11}\right) \partial_{y_{1}} g_{1}\left(k_{s}\right)
\end{array}\right)
$$

Let $F_{1}=\operatorname{diag}\left(\partial_{y_{1}} g_{1}\left(k_{1}\right), \ldots, \partial_{y_{1}} g_{1}\left(k_{s}\right)\right)$ and $F_{2}=\operatorname{diag}\left(\partial_{y_{2}} g_{2}\left(k_{1}\right), \ldots, \partial_{y_{2}} g_{2}\left(k_{s}\right)\right)$. The above result can be simplified as

$$
\begin{aligned}
& \left|I_{s} \otimes I-h(A \otimes I . * E(h K)) F\right| \\
= & \left|I_{s} \otimes I-h\left(A \otimes I . * E\left(h K_{11}\right)\right) F_{1}\right|\left|I_{s} \otimes I-h\left(A \otimes I . * E\left(h K_{22}\right)\right) F_{2}\right| .
\end{aligned}
$$

Since the SSEI method is of volume preservation for the vector field $u\left(y_{1}\right)$, the following condition is true

$$
\left|I_{s} \otimes I-h\left(A \otimes I . * E\left(h K_{11}\right)\right) F_{1}\right|=\left|e^{h K_{11}}\right|\left|I_{s} \otimes I+h\left(A^{\top} \otimes I . * E\left(h K_{11}\right)\right) F_{1}\right| .
$$


On the other hand, we compute

$$
\begin{aligned}
& \left|I_{s} \otimes I-h\left(A \otimes I . * E\left(h K_{22}\right)\right) F_{2}\right| \\
= & \left|\left(I_{s} \otimes P\right)\left(I_{s} \otimes P^{-1}\right)-h\left(I_{s} \otimes P\right)\left(A \otimes I . * E\left(h K_{22}\right)\right)\left(I_{s} \otimes P^{-1}\right)\left(I_{s} \otimes P\right) F\left(I_{s} \otimes P^{-1}\right)\right| \\
= & \left|I_{s} \otimes I+h\left(I_{s} \otimes P\right)\left(A \otimes I . * E\left(h K_{22}\right)\right)\left(I_{s} \otimes P^{-1}\right) F_{2}^{\top}\right| \\
= & \left|I_{s} \otimes I+h\left(A \otimes I * * E\left(-h K_{22}^{\top}\right)\right) F_{2}^{\top}\right| \\
= & \left|I_{s} \otimes I+h F_{2}\left(A^{\top} \otimes I . * E\left(-h K_{22}^{\top}\right)^{\top}\right)\right| \text { (transpose) } \\
= & \mid I_{s} \otimes I+h\left(A^{\top} \otimes I . * E\left(-h K_{22}^{\top}\right) F_{2} \mid\right. \text { (Sylvester's law) } \\
= & \left.\left|I_{s} \otimes I+h\left(A^{\top} \otimes I . * E\left(h K_{22}\right)\right) F_{2}\right| \text { (property (14) }\right) .
\end{aligned}
$$

Therefore, the VP condition (10) holds and the SSEI method is of volume preservation for vector fields in $\mathcal{F}^{(\infty)}$.

\subsection{Vector field $\mathcal{F}^{(2)}$}

Suppose that the function $f(y)$ of (11) falls into $\mathcal{F}^{(2)}$. Under this situation, (17) and (18) are still true. We obtain the following result about the VP property of SSEI methods.

Theorem 4.7 Consider a one-stage or two-stage SSEI with $c_{1}=c_{2}$ (or a composition of such method) that is of volume preservation for the vector field $u\left(y_{1}\right): \mathbb{R}^{m} \rightarrow \mathbb{R}^{m}$. Letting $v\left(y_{1}, y_{2}\right)$ : $\mathbb{R}^{m+n} \rightarrow \mathbb{R}^{m+n}$, we assume that there exists an invertible matrix $P$ such that for all $y_{1}, y_{2}$,

$$
P \partial_{y_{2}} v\left(y_{1}, y_{2}\right) P^{-1}=-\partial_{y_{2}} v\left(y_{1}, y_{2}\right), \quad P \partial_{y_{2}} g_{2}\left(y_{1}, y_{2}\right) P^{-1}=-\partial_{y_{2}} g_{2}\left(y_{1}, y_{2}\right) .
$$

Then the SSEI method is of volume preservation for the vector fields $f\left(y_{1}, y_{2}\right)=\left(u\left(y_{1}\right), v\left(y_{1}, y_{2}\right)\right)^{\top}$ in $\mathcal{F}^{(2)}$.

$\underline{\text { Proof }}$ From the conditions of this theorem, it follows that $P K_{22} P^{-1}=-K_{22}$ and $\left|e^{h K_{22}}\right|=1$.

For the one-stage SSEI, the condition for volume preservation is

$$
\left|I-h a_{11} g^{\prime}\left(k_{1}\right)\right|=\left|I+h a_{11} g^{\prime}\left(k_{1}\right)\right|,
$$

which can be rewritten as

$$
\left|I-h a_{11} \partial_{y_{1}} g_{1}\right|\left|I-h a_{11} \partial_{y_{2}} g_{2}\right|=\left|I+h a_{11} \partial_{y_{1}} g_{1}\right|\left|I+h a_{11} \partial_{y_{2}} g_{2}\right| .
$$

Since the method is of volume preservation for the vector field $u\left(y_{1}\right)$, we have

$$
\left|I-h a_{11} \partial_{y_{1}} g_{1}\right|=\left|I+h a_{11} \partial_{y_{1}} g_{1}\right| .
$$

On the other hand,

$$
\left|I-h a_{11} \partial_{y_{2}} g_{2}\right|=\left|P P^{-1}-h a_{11} P \partial_{y_{2}} g_{2} P^{-1}\right|=\left|I+h a_{11} \partial_{y_{2}} g_{2}\right| .
$$

Thus (19) is proved. 
For the two-stage SSEI, it is of volume preservation if and only if (15) is true. According to the special result of $g^{\prime}$, one has

$$
\begin{gathered}
\text { the left hand side of } 1 \text { (15) } \\
=\mid I-h a_{11} \partial_{y_{1}} g_{1}\left(k_{1}\right)-h a_{22} \partial_{y_{1}} g_{1}\left(k_{2}\right)+h^{2} a_{11} a_{22} \partial_{y_{1}} g_{1}\left(k_{1}\right) \partial_{y_{1}} g_{1}\left(k_{2}\right) \\
\quad-h^{2} a_{12} a_{21} \partial_{y_{1}} g_{1}\left(k_{2}\right) \partial_{y_{1}} g_{1}\left(k_{1}\right) \mid \\
\mid I-h a_{11} \partial_{y_{2}} g_{2}\left(k_{1}\right)-h a_{22} \partial_{y_{2}} g_{2}\left(k_{2}\right)+h^{2} a_{11} a_{22} \partial_{y_{2}} g_{2}\left(k_{1}\right) \partial_{y_{2}} g_{2}\left(k_{2}\right) \\
\quad-h^{2} a_{12} a_{21} \partial_{y_{2}} g_{2}\left(k_{2}\right) \partial_{y_{2}} g_{2}\left(k_{1}\right) \mid \\
=\quad \mid I+h a_{11} \partial_{y_{1}} g_{1}\left(k_{1}\right)+h a_{22} \partial_{y_{1}} g_{1}\left(k_{2}\right)+h^{2} a_{11} a_{22} \partial_{y_{1}} g_{1}\left(k_{1}\right) \partial_{y_{1}} g_{1}\left(k_{2}\right) \\
\quad-h^{2} a_{12} a_{21} \partial_{y_{1}} g_{1}\left(k_{2}\right) \partial_{y_{1}} g_{1}\left(k_{1}\right) \mid \\
\mid I-h a_{11} \partial_{y_{2}} g_{2}\left(k_{1}\right)-h a_{22} \partial_{y_{2}} g_{2}\left(k_{2}\right)+h^{2} a_{11} a_{22} \partial_{y_{2}} g_{2}\left(k_{1}\right) \partial_{y_{2}} g_{2}\left(k_{2}\right) \\
\quad-h^{2} a_{12} a_{21} \partial_{y_{2}} g_{2}\left(k_{2}\right) \partial_{y_{2}} g_{2}\left(k_{1}\right) \mid .
\end{gathered}
$$

It then can be verified that

$$
\begin{aligned}
& \mid I-h a_{11} \partial_{y_{2}} g_{2}\left(k_{1}\right)-h a_{22} \partial_{y_{2}} g_{2}\left(k_{2}\right)+h^{2} a_{11} a_{22} \partial_{y_{2}} g_{2}\left(k_{1}\right) \partial_{y_{2}} g_{2}\left(k_{2}\right) \\
& -h^{2} a_{12} a_{21} \partial_{y_{2}} g_{2}\left(k_{2}\right) \partial_{y_{2}} g_{2}\left(k_{1}\right) \mid \\
& =\mid P P^{-1}-h a_{11} P \partial_{y_{2}} g_{2}\left(k_{1}\right) P^{-1}-h a_{22} P \partial_{y_{2}} g_{2}\left(k_{2}\right) P^{-1}+h^{2} a_{11} a_{22} P \partial_{y_{2}} g_{2}\left(k_{1}\right) P^{-1} \\
& P \partial_{y_{2}} g_{2}\left(k_{2}\right) P^{-1}-h^{2} a_{12} a_{21} P \partial_{y_{2}} g_{2}\left(k_{2}\right) P^{-1} P \partial_{y_{2}} g_{2}\left(k_{1}\right) P^{-1} \mid \\
& =\mid I+h a_{11} \partial_{y_{2}} g_{2}\left(k_{1}\right)+h a_{22} \partial_{y_{2}} g_{2}\left(k_{2}\right)+h^{2} a_{11} a_{22} \partial_{y_{2}} g_{2}\left(k_{1}\right) \partial_{y_{2}} g_{2}\left(k_{2}\right) \\
& \quad-h^{2} a_{12} a_{21} \partial_{y_{2}} g_{2}\left(k_{2}\right) \partial_{y_{2}} g_{2}\left(k_{1}\right) \mid .
\end{aligned}
$$

Consequently,

$$
\begin{gathered}
\text { the left hand side of (15) } \\
=\quad \mid I+h a_{11} \partial_{y_{1}} g_{1}\left(k_{1}\right)+h a_{22} \partial_{y_{1}} g_{1}\left(k_{2}\right)+h^{2} a_{11} a_{22} \partial_{y_{1}} g_{1}\left(k_{1}\right) \partial_{y_{1}} g_{1}\left(k_{2}\right) \\
\quad-h^{2} a_{12} a_{21} \partial_{y_{1}} g_{1}\left(k_{2}\right) \partial_{y_{1}} g_{1}\left(k_{1}\right) \mid \\
\mid I+h a_{11} \partial_{y_{2}} g_{2}\left(k_{1}\right)+h a_{22} \partial_{y_{2}} g_{2}\left(k_{2}\right)+h^{2} a_{11} a_{22} \partial_{y_{2}} g_{2}\left(k_{1}\right) \partial_{y_{2}} g_{2}\left(k_{2}\right) \\
\quad-h^{2} a_{12} a_{21} \partial_{y_{2}} g_{2}\left(k_{2}\right) \partial_{y_{2}} g_{2}\left(k_{1}\right) \mid \\
=\quad \text { the right hand side of (15). }
\end{gathered}
$$

Therefore, all two-stage SSEI methods with $c_{1}=c_{2}$ are of volume preservation.

Remark 4.8 We note that when $K=\mathbf{0}$, all the results given in this section reduce to those proposed in [1], which demonstrate the wilder applications of the analysis.

\section{Applications to various problems}

In this section, we pay attention to the application of the SSEI methods to various problems and show the volume preservation of different exponential integrators and ERKN/RKN methods by using the analysis given in Section 4.

\subsection{Highly oscillatory second-order systems}

Consider the following first-order systems

$$
y^{\prime}(t)=J^{-1} M y(t)+J^{-1} \nabla V(y(t)),
$$


where the matrix $J$ is constant and invertible, $M$ is a symmetric matrix and $V$ is a differentiable function. This system is the exact pattern (11) with

$$
K=J^{-1} M, \quad g(y(t))=J^{-1} \nabla V(y(t)) .
$$

It can be verified that

$$
J g^{\prime}(y) J^{-1}=J J^{-1} \nabla^{2} V(y) J^{-1}=\nabla^{2} V(y) J^{-1}=-g^{\prime}(y)^{\top}
$$

and

$$
J\left(K+g^{\prime}(y)\right) J^{-1}=-\left(K+g^{\prime}(y)\right)^{\top} .
$$

This shows that the set $\mathcal{H}$ contains all vector fields of (20) with the same $P=J$. Thus in the light of Theorem 4.3, all SSEI methods are of volume preservation for solving the system (20).

When $J=\left(\begin{array}{cc}0 & I \\ -I & 0\end{array}\right)$, the system (20) is a Hamiltonian system $y^{\prime}(t)=J^{-1} \nabla H(y(t))$ with the Hamiltonian $H(y)=\frac{1}{2} y^{\top} M y+V(y)$. All SSEI methods are of volume preservation for this Hamiltonian system. This is another explanation of the fact that symplectic exponential integrators are of volume preservation for Hamiltonian systems.

Consider another special and important case of (20) by choosing

$$
y=\left(\begin{array}{c}
q \\
p
\end{array}\right), \quad J^{-1}=\left(\begin{array}{cc}
0 & I \\
-I & N
\end{array}\right), \quad M=\left(\begin{array}{cc}
\Omega & 0 \\
0 & I
\end{array}\right), \quad V(y)=V_{1}(q)
$$

which gives the following second-order ODE

$$
q^{\prime \prime}-N q^{\prime}+\Omega q=-\nabla V_{1}(q)
$$

This system stands for highly oscillatory problems and many problems fall into this kind of equation such as the dissipative molecular dynamics, the (damped) Duffing, charged-particle dynamics in a constant magnetic field and semidiscrete nonlinear wave equations. Applying the SSEI methods to (22) and considering Theorem 4.3 we obtain the following corollary.

Corollary 5.1 The following s-stage adapted exponential integrator

$$
\left\{\begin{array}{c}
k_{i}=\exp ^{11}\left(c_{i} h K\right) q_{n}+\exp ^{12}\left(c_{i} h K\right) q_{n}^{\prime}-h \sum_{j=1}^{s} a_{i j} \exp ^{12}\left(\left(c_{i}-c_{j}\right) h K\right) \nabla V_{1}\left(k_{j}\right), \\
i=1,2, \ldots, s, \\
q_{n+1}=\exp ^{11}(h K) q_{n}+\exp ^{12}(h K) q_{n}^{\prime}-h \sum_{i=1}^{s} b_{i} \exp ^{12}\left(\left(1-c_{i}\right) h K\right) \nabla V_{1}\left(k_{i}\right), \\
q_{n+1}^{\prime}=\exp ^{21}(h K) q_{n}+\exp ^{22}(h K) q_{n}^{\prime}-h \sum_{i=1}^{s} b_{i} \exp ^{22}\left(\left(1-c_{i}\right) h K\right) \nabla V_{1}\left(k_{i}\right)
\end{array}\right.
$$

are of volume preservation for the second-order highly oscillatory equation (22), where $\exp (h K)$ is partitioned into $\left(\begin{array}{ll}\exp ^{11}(h K) & \exp ^{12}(h K) \\ \exp ^{21}(h K) & \exp ^{22}(h K)\end{array}\right)$ and the same denotations are used for other matrixvalued functions. Here $c=\left(c_{1}, \ldots, c_{s}\right)^{\top}, b=\left(b_{1}, \ldots, b_{s}\right)^{\top}$ and $A=\left(a_{i j}\right)_{s \times s}$ are given in Definition 
2.2. If $N$ commutes with $\Omega$, the results of $\exp ^{i j}$ for $i, j=1,2$ appearing in (23) can be expressed explicitly. After some calculations, it is obtained that

$$
\begin{aligned}
& \exp ^{11}(h K)=e^{\frac{h}{2} N}\left(\cosh \left(\frac{h}{2} \sqrt{N^{2}-4 \Omega}\right)-N \sinh \left(\frac{h}{2} \sqrt{N^{2}-4 \Omega}\right)\left(\sqrt{N^{2}-4 \Omega}\right)^{-1}\right), \\
& \exp ^{12}(h K)=2 e^{\frac{h}{2} N} \sinh \left(\frac{h}{2} \sqrt{N^{2}-4 \Omega}\right)\left(\sqrt{N^{2}-4 \Omega}\right)^{-1} \\
& \exp ^{21}(h K)=-\Omega \exp ^{12}(h K) \\
& \exp ^{22}(h K)=e^{\frac{h}{2} N}\left(\cosh \left(\frac{h}{2} \sqrt{N^{2}-4 \Omega}\right)+N \sinh \left(\frac{h}{2} \sqrt{N^{2}-4 \Omega}\right)\left(\sqrt{N^{2}-4 \Omega}\right)^{-1}\right) .
\end{aligned}
$$

The results are still true if we replace $h$ by $k h$ with any $k \in \mathbb{R}$.

If we further assume that $\Omega=0$, the equation (22) becomes

$$
q^{\prime \prime}=N q^{\prime}-\nabla V_{1}(q)
$$

One typical example of this system is charged-particle dynamics in a constant magnetic field, which can be expressed by (see [11])

$$
x^{\prime \prime}=x^{\prime} \times B+F(x) .
$$

Here $x(t) \in \mathbb{R}^{3}$ describes the position of a particle moving in an electro-magnetic field, $F(x)=$ $-\nabla_{x} U(x)$ is an electric field with the scalar potential $U(x)$, and $B=\nabla_{x} \times A(x)$ is a constant magnetic field with the vector potential $A(x)=-\frac{1}{2} x \times B$.

Under the condition that $\Omega=0$, formulae (24) can be written more succinctly as:

$$
\exp ^{11}(h K)=I, \quad \exp ^{12}(h K)=h \varphi_{1}(h N), \quad \exp ^{21}(h K)=0, \quad \exp ^{22}(h K)=\varphi_{0}(h N),
$$

where the $\varphi$-functions are defined by (see [16, 17])

$$
\varphi_{0}(z)=e^{z}, \quad \varphi_{k}(z)=\int_{0}^{1} e^{(1-\sigma) z} \frac{\sigma^{k-1}}{(k-1) !} d \sigma, \quad k=1,2, \ldots
$$

We then get the following volume preserving methods for the special and important second-order system (25).

Corollary 5.2 The following s-stage integrator

$$
\left\{\begin{array}{l}
k_{i}=q_{n}+c_{i} h \varphi_{1}\left(c_{i} h N\right) q_{n}^{\prime}-h^{2} \sum_{j=1}^{s} a_{i j}\left(c_{i}-c_{j}\right) \varphi_{1}\left(\left(c_{i}-c_{j}\right) h N\right) \nabla V_{1}\left(k_{j}\right), \\
q_{n+1}=q_{n}+h \varphi_{1}(h N) q_{n}^{\prime}-h^{2} \sum_{i=1}^{s} b_{i}\left(1-c_{i}\right) \varphi_{1}\left(\left(1-c_{i}\right) h N\right) \nabla V_{1}\left(k_{i}\right), \\
q_{n+1}^{\prime}=\varphi_{0}(h N) q_{n}^{\prime}-h \sum_{i=1}^{s} b_{i} \varphi_{0}\left(\left(1-c_{i}\right) h N\right) \nabla V_{1}\left(k_{i}\right)
\end{array}\right.
$$

are of volume preservation for the highly oscillatory second-order system (25), where $c=\left(c_{1}, \ldots, c_{s}\right)^{\top}, b=$ $\left(b_{1}, \ldots, b_{s}\right)^{\top}$ and $A=\left(a_{i j}\right)_{s \times s}$ are given in Definition [2.2.

Remark 5.3 It is noted that the above two corollaries are new discoveries which are of great importance to Geometric Integration for second-order highly oscillatory problems. 


\subsection{Separable partitioned systems}

It has been proved in 1 that the set $\mathcal{S}$ contains all separable partitioned systems. As an example, we consider

$$
\left(\begin{array}{c}
q \\
p
\end{array}\right)^{\prime}=\left(\begin{array}{c}
p \\
-\Omega q+\tilde{g}(q)
\end{array}\right)=\left(\begin{array}{cc}
0 & I \\
-\Omega & 0
\end{array}\right)\left(\begin{array}{c}
q \\
p
\end{array}\right)+\left(\begin{array}{c}
0 \\
\tilde{g}(q)
\end{array}\right)
$$

which is exactly the system (10) with

$$
K=\left(\begin{array}{cc}
0 & I \\
-\Omega & 0
\end{array}\right), g=\left(\begin{array}{c}
0 \\
g(q)
\end{array}\right), f=\left(\begin{array}{c}
p \\
-\Omega q+\tilde{g}(q)
\end{array}\right)
$$

It can be checked that $f$ and $g$ both fall into $\mathcal{S}$ with the same $P=\operatorname{diag}(I,-I)$. For this special matrix $K$, it is clear that

$$
e^{x K}=\left(\begin{array}{cc}
\phi_{0}\left(x^{2} \Omega\right) & x \phi_{1}\left(x^{2} \Omega\right) \\
-x \Omega \phi_{1}\left(x^{2} \Omega\right) & \phi_{0}\left(x^{2} \Omega\right)
\end{array}\right), \quad \text { for } \quad x \in \mathbb{R}
$$

with $\phi_{i}(\Omega):=\sum_{l=0}^{\infty} \frac{(-1)^{l} \Omega^{l}}{(2 l+i) !}$ for $i=0,1$. Thus the exponential integrator (3) has a special form, which is called as extended RKN (ERKN) integrators and is represented below.

Definition 5.4 (See 34]) An s-stage ERKN integrator for solving (29) is defined by

$$
\left\{\begin{aligned}
Q_{i} & =\phi_{0}\left(c_{i}^{2} V\right) q_{n}+h c_{i} \phi_{1}\left(c_{i}^{2} V\right) p_{n}+h^{2} \sum_{j=1}^{s} \bar{a}_{i j}(V) \tilde{g}\left(Q_{j}\right), \quad i=1, \ldots, s \\
q_{n+1} & =\phi_{0}(V) q_{n}+h \phi_{1}(V) p_{n}+h^{2} \sum_{i=1}^{s} \bar{b}_{i}(V) \tilde{g}\left(Q_{i}\right) \\
p_{n+1} & =-h \Omega \phi_{1}(V) q_{n}+\phi_{0}(V) p_{n}+h \sum_{i=1}^{s} b_{i}(V) \tilde{g}\left(Q_{i}\right)
\end{aligned}\right.
$$

where $c_{i}$ for $i=1, \ldots, s$ are real constants, $b_{i}(V), \bar{b}_{i}(V)$ for $i=1, \ldots, s$, and $\bar{a}_{i j}(V)$ for $i, j=$ $1, \ldots, s$ are matrix-valued functions of $V \equiv h^{2} \Omega$.

ERKN integrators were firstly proposed in 34. Further discussions about ERKN integrators have been given recently, including symmetric integrators (see 30]), symplectic integrators (see [33]), energy-preserving integrators (see [32]) and other kinds integrators (see [26, 28]). However, to our knowledge, the volume-preserving property of ERKN integrators has not been researched yet in the literature. With the analysis given in this paper, we get the following VP result of ERKN integrators.

Corollary 5.5 Consider a kind of s-stage ERKN integrators

$$
\left\{\begin{array}{c}
Q_{i}=\phi_{0}\left(c_{i}^{2} V\right) q_{n}+h c_{i} \phi_{1}\left(c_{i}^{2} V\right) p_{n}+h^{2} \sum_{j=1}^{s} a_{i j}\left(c_{i}-c_{j}\right) \phi_{1}\left(\left(c_{i}-c_{j}\right)^{2} V\right) \tilde{g}\left(Q_{j}\right), \\
i=1, \ldots, s \\
q_{n+1}=\phi_{0}(V) q_{n}+h \phi_{1}(V) p_{n}+h^{2} \sum_{i=1}^{s} b_{i}\left(1-c_{i}\right) \phi_{1}\left(\left(1-c_{i}\right)^{2} V\right) \tilde{g}\left(Q_{i}\right) \\
p_{n+1}=-h \Omega \phi_{1}(V) q_{n}+\phi_{0}(V) p_{n}+h \sum_{i=1}^{s} b_{i} \phi_{0}\left(\left(1-c_{i}\right)^{2} V\right) \tilde{g}\left(Q_{i}\right)
\end{array}\right.
$$


where $c=\left(c_{1}, \ldots, c_{s}\right)^{\top}, \quad b=\left(b_{1}, \ldots, b_{s}\right)^{\top}$ and $A=\left(a_{i j}\right)_{s \times s}$ are given in Definition 2.2. Under the condition that $b_{j} \neq 0$ for all $j=1, \ldots, s$, all one-stage and two-stage (with $c_{1}=c_{2}$ ) ERKN integrators (31), and compositions thereof, are of volume preservation for solving the separable partitioned system (29).

Proof In the light of Definition 2.2 and the result (30), we adapt the SSEI methods to the system (29) and then get the scheme (31). Based on Theorem 4.4, the volume preserving result of (31) is immediately obtained.

Remark 5.6 We note that this is a novel result which studies the volume-preserving ERKN integrators for (29). Moreover, from the scheme (31), it can be observed that all one-stage and two-stage with $c_{1}=c_{2}$ ERKN integrators are explicit, which means that we obtain explicit volume preserving ERKN integrators for the separable partitioned system (29).

If $\Omega$ is a symmetric and positive semi-definite matrix, and $\tilde{g}(q)=-\nabla U(q)$, the system (29) is an oscillatory Hamiltonian system $\left(\begin{array}{l}q \\ p\end{array}\right)^{\prime}=\left(\begin{array}{cc}0 & I \\ -I & 0\end{array}\right) \nabla H(q, p)$ with the Hamiltonian

$$
H(q, p)=\frac{1}{2} p^{\top} p+\frac{1}{2} q^{\top} \Omega q+U(q) .
$$

It has been noted in Subsection 5.1 that this vector field falls into the set $\mathcal{H}$. Thus Theorem 4.3 provides another way to prove the well-known fact that all symplectic ERKN integrators (31) are of volume preservation for the oscillatory Hamiltonian system (32).

In what follows, we study the volume-preserving property of RKN methods for second-order ODEs. Consider $\Omega=0$ for the above analysis and under this situation, ERKN integrators reduce to RKN methods. Therefore, we are now in a position to present the following volume-preserving property for RKN methods.

Corollary 5.7 Consider the following s-stage RKN methods

$$
\left\{\begin{array}{l}
Q_{i}=q_{n}+h c_{i} q_{n}^{\prime}+h^{2} \sum_{j=1}^{s} a_{i j}\left(c_{i}-c_{j}\right) \tilde{g}\left(Q_{j}\right), \quad i=1, \ldots, s \\
q_{n+1}=q_{n}+h q_{n}^{\prime}+h^{2} \sum_{i=1}^{s} b_{i}\left(1-c_{i}\right) \tilde{g}\left(Q_{i}\right) \\
q_{n+1}^{\prime}=q_{n}^{\prime}+h \sum_{i=1}^{s} b_{i} \tilde{g}\left(Q_{i}\right)
\end{array}\right.
$$

with the coefficients $c=\left(c_{1}, \ldots, c_{s}\right)^{\top}, b=\left(b_{1}, \ldots, b_{s}\right)^{\top}$ and $A=\left(a_{i j}\right)_{s \times s}$ of an s-stage $R K$ method. If this $R K$ method is symplectic and $b_{j} \neq 0$ for all $j=1, \ldots, s$, all one-stage and two-stage $R K N$ methods (33) (and compositions thereof) are of volume preservation for solving the second-order system $q^{\prime \prime}=\tilde{g}(q)$.

Remark 5.8 It is noted that the fact of this corollary can be derived in a different way. Hairer, Lubich and Wanner have proved in [13] that any symplectic RK method with at most two stages (and any composition of such methods) is volume preserving for separable divergence free vector fields. Rewriting the second-order equation $q^{\prime \prime}=\tilde{g}(q)$ as a first-order system and applying symplectic $R K$ methods to it implies the result of Corollary 5.7. It is seen that the analysis of volume-preserving ERKN integrators provides an alternative derivation of the volume preservation of RKN methods. 


\subsection{Other applications}

It has been shown in 1 that $\mathcal{F}^{(\infty)}$ contains the affine vector fields $f(y)=L y+d$ such that $\left|I+\frac{h}{2} L\right|=\left|I-\frac{h}{2} L\right|$ for all $h>0$. For solving the system in the affine vector fields, the exponential integrator (3) becomes

$$
\left\{\begin{array}{l}
k_{i}=e^{c_{i} h L} y_{n}+h \sum_{j=1}^{s} \bar{a}_{i j}(h L) d, \quad i=1,2, \ldots, s \\
y_{n+1}=e^{h L} y_{n}+h \sum_{i=1}^{s} \bar{b}_{i}(h L) d .
\end{array}\right.
$$

In the light of Theorem 4.6 this SSEI method is of volume preservation for the affine vector fields.

It was also noted in [1] that $\mathcal{F}^{(\infty)}$ contains the vector fields $f(y)$ such that $f^{\prime}(y)=J S(y)$ with a skew-symmetric matrix $J$ and the symmetric matrix $S(y)$. Assume that

$$
K=J M, \quad g^{\prime}(y)=J S(y),
$$

where $M$ is a symmetric matrix. The system (11) with the vector field (34) falls into $\mathcal{F}^{(\infty)}$. Thus all SSEI methods are of volume preservation for the vector field (34).

\section{$6 \quad$ Numerical examples}

In order to show the performance of SSEI methods, the solvers chosen for comparison are:

- SSRK1: the Gauss-Legendre method of order two;

- SSEI1: the one-stage SSEI method with the coefficients (4), where the RK method (5) is chosen as SSRK1;

- SSRK2: the Gauss-Legendre method of order four;

- SSEI2: the two-stage SSEI method with the coefficients (4), where the RK method (5) is chosen as SSRK2.

It is noted that all these methods are general implicit and we use fixed-point iteration here. We set $10^{-16}$ as the error tolerance and 100 as the maximum number of each fixed-point iteration.

Problem 1. As the first numerical example, we consider the Duffing equation defined by

$$
\left(\begin{array}{l}
q \\
p
\end{array}\right)^{\prime}=\left(\begin{array}{cc}
0 & 1 \\
-\omega^{2}-k^{2} & 0
\end{array}\right)\left(\begin{array}{l}
q \\
p
\end{array}\right)+\left(\begin{array}{c}
0 \\
2 k^{2} q^{3}
\end{array}\right), \quad\left(\begin{array}{c}
q(0) \\
p(0)
\end{array}\right)=\left(\begin{array}{c}
0 \\
\omega
\end{array}\right) .
$$

The exact solution of this system is $q(t)=s n(\omega t ; k / \omega)$ with the Jacobi elliptic function $s n$. Since it is a Hamiltonian system, all the methods chosen for comparison are volume preserving. For this problem, we choose $k=0.07$ and $\omega=20$ and then solve it on the interval $[0,100]$ with different stepsizes $h=1 / 2,1 / 10,1 / 50,1 / 200$. The numerical flows at the time points $\left\{\frac{1}{2} i\right\}_{i=1, \ldots, 200}$ of the four methods are given in Figure 2, From the results, it can be observed clearly that the integrators SSEI1 and SSEI2 perform better than Runge-Kutta methods in the preservation of volume. Finally, we integrate this problem in $\left[0, t_{\text {end }}\right]$ with $h=0.1 / 2^{i}$ for $i=1, \ldots, 4$. The relative global errors 

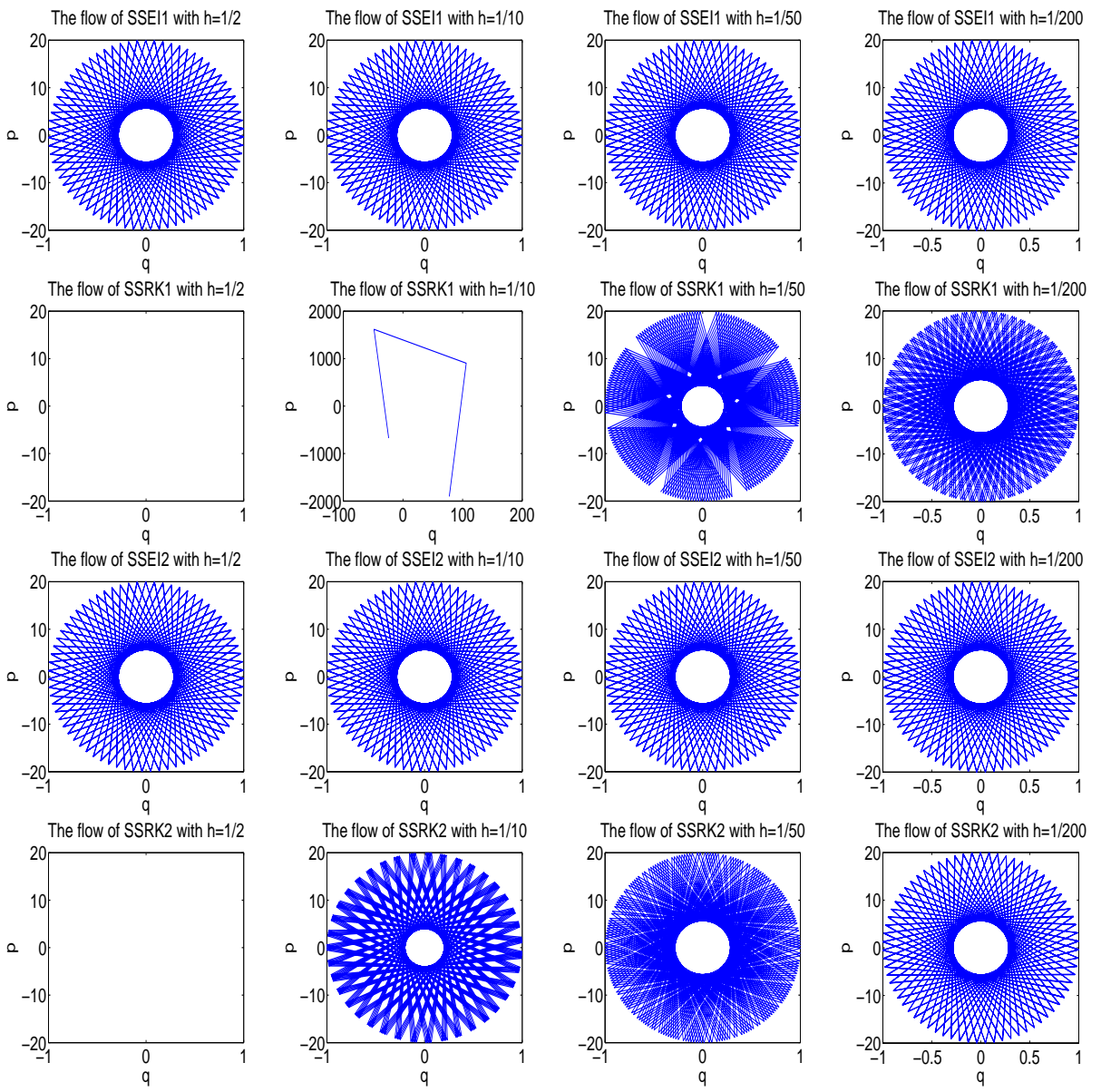

Figure 2: Problem 1: the flows of different methods.
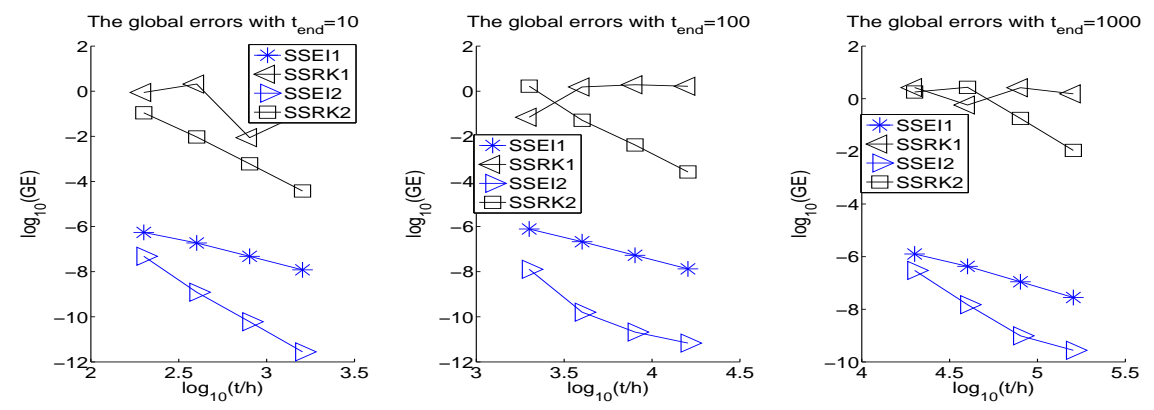

Figure 3: Problem 1: the relative global errors. 
for different $t_{\text {end }}$ are presented in Figure 3 These results show clearly again that exponential integrators have better accuracy than Runge-Kutta methods.

Problem 2. Consider the following divergence free ODEs

$$
\left(\begin{array}{l}
x \\
y \\
z
\end{array}\right)^{\prime}=\left(\begin{array}{ccc}
0 & -\omega & 0 \\
\omega & 0 & -\omega \\
0 & \omega & 0
\end{array}\right)\left(\begin{array}{l}
x \\
y \\
z
\end{array}\right)+\left(\begin{array}{c}
\sin (x-z) \\
0 \\
\sin (x-z)
\end{array}\right)
$$

By choosing $P=\left(\begin{array}{lll}0 & 0 & 1 \\ 0 & 1 & 0 \\ 1 & 0 & 0\end{array}\right)$, it can be checked that the vector field of this problem falls into $\mathcal{S}$. We consider $\omega=100$ and $(0.5,0.5,0.5)^{\top}$ as the initial value. This problem is firstly integrated on $[0,100]$ with $h=1 / 50,1 / 100,1 / 200,1 / 400$ and the numerical flows $x$ and $y$ at the time points $\left\{\frac{1}{2} i\right\}_{i=1, \ldots, 200}$ are shown in Figure4 Then the relative global errors for different $t_{\text {end }}$ with $h=0.1 / 2^{i}$ for $i=2, \ldots, 5$ are given in Figure 5 . These results demonstrate clearly again that SSEI methods perform better than SSRK methods.

Problem 3. Consider the damped Helmholtz-Duffing oscillator (see [6])

$$
q^{\prime \prime}+2 v q^{\prime}+A q=-B q^{2}-\varepsilon q^{3},
$$

where $q$ denotes the displacement of the system, $A$ is the natural frequency, $\varepsilon$ is a non-linear system parameter, $v$ is the damping factor, and $B$ is a system parameter independent of time. It is well known that the dynamical behavior of eardrum oscillations, elasto-magnetic suspensions, thin laminated plates, graded beams, and other physical phenomena all fall into this kind of equations. We choose the parameters

$$
v=0.01, A=200, B=-0.5, \varepsilon=1
$$

and the initial value $q(0)=1$ and $q^{\prime}(0)=15.199$. This problem is firstly integrated on $[0,200]$ with $h=1 / 2,1 / 10,1 / 50,1 / 200$. We present the numerical flows $q$ and $p=q^{\prime}$ at the time points $\left\{\frac{1}{2} i\right\}_{i=1, \ldots, 400}$ in Figure 6. We then solve the problem with different $t_{\text {end }}=10,100,1000$ and $h=0.1 / 2^{i}$ for $i=0, \ldots, 3$. The relative global errors are shown in Figure 7 It follows again from the results that SSEI methods perform much better than SSRK methods.

Problem 4. The last numerical experiment is concerned with the charged particle system with a constant magnetic field (see [11]). The system can be given by (26) with the potential $U(x)=$ $\frac{1}{100 \sqrt{x_{1}^{2}+x_{2}^{2}}}$ and the constant magnetic field $B=(0,0,10)^{\top}$. The initial values are chosen as $x(0)=$ $(0.7,1,0.1)^{\top}$ and $x^{\prime}(0)=(0.9,0.5,0.4)^{\top}$. We firstly integrate this system on $[0,100]$ with $h=$ $1 / 2,1 / 10,1 / 50,1 / 200$ and show the numerical flows $x_{2}$ and $v_{2}=x_{2}^{\prime}$ at the time points $\left\{\frac{1}{2} i\right\}_{i=1, \ldots, 200}$ in Figure 8 Then the problem is solved with $t_{\text {end }}=10,100,1000$ and $h=0.1 / 2^{i}$ for $i=0, \ldots, 3$ and the relative global errors are shown in Figure 9. The SSEI methods are also shown to be robust to this problem. Here, it is important to note that our SSEI1 method is explicit (see (28)) when applied to this problem, whereas, the SSRK1 method is implicit and the iteration is required for solving this problem. This fact shows another advantage of our volume-preserving exponential integrators in comparison with volume-preserving RK methods. 

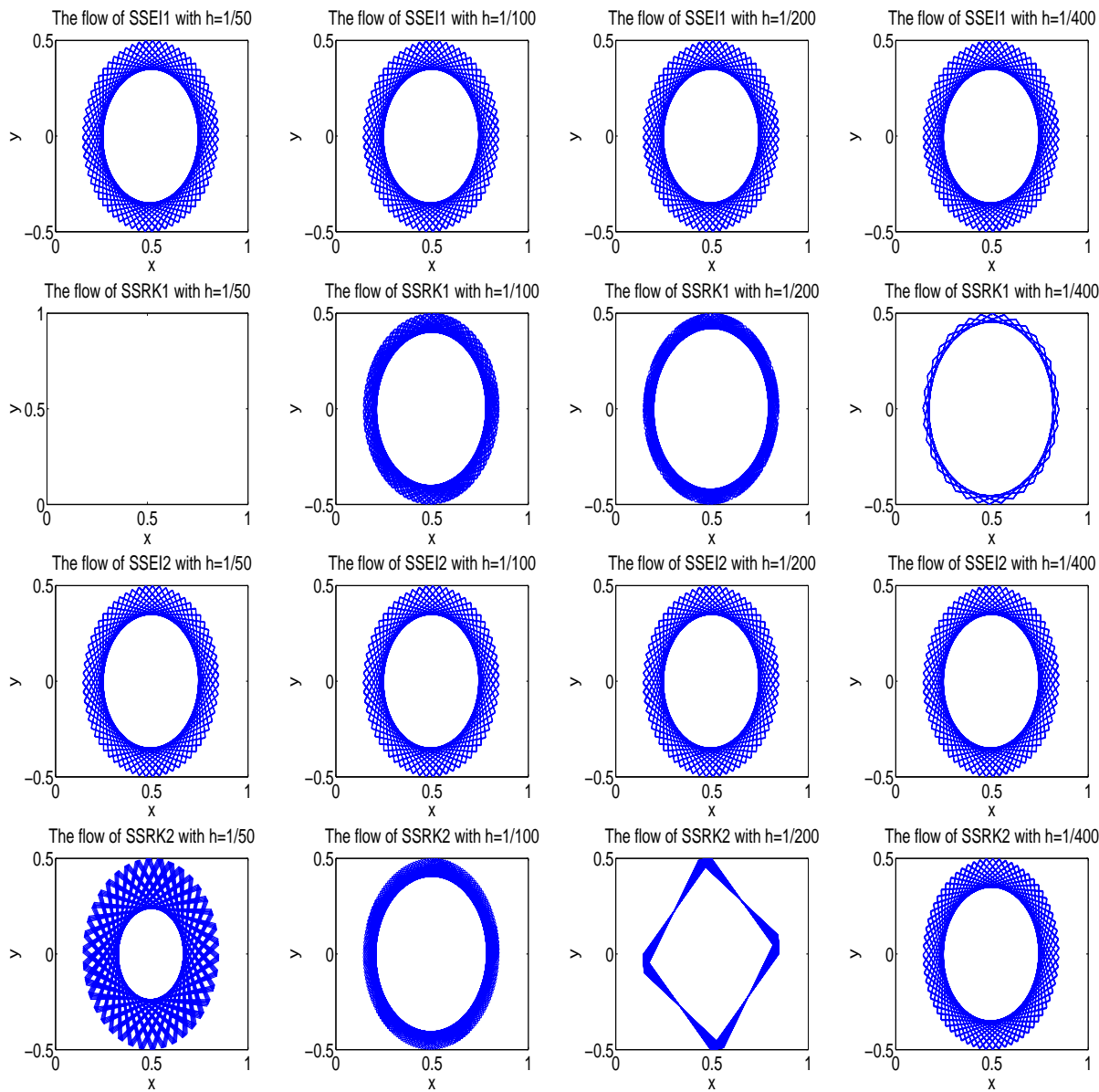

Figure 4: Problem 2: the flows of different methods.
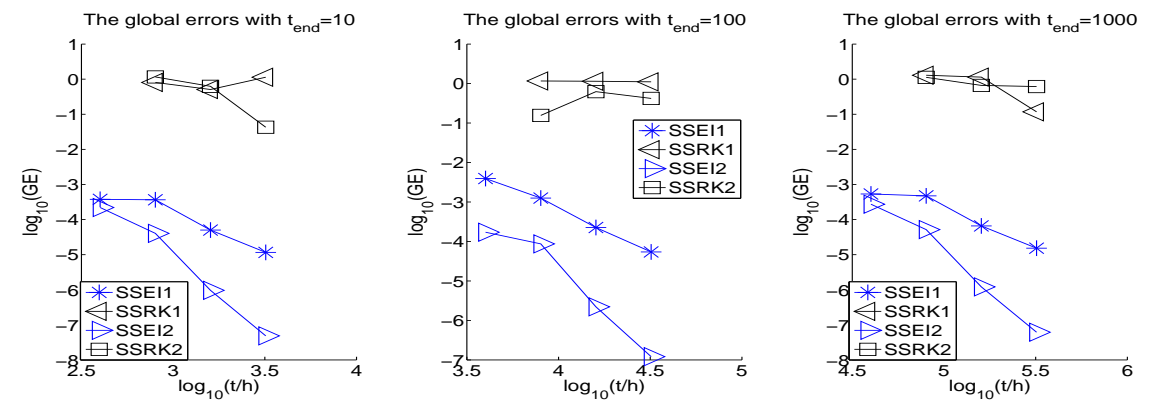

Figure 5: Problem 2: the relative global errors. 

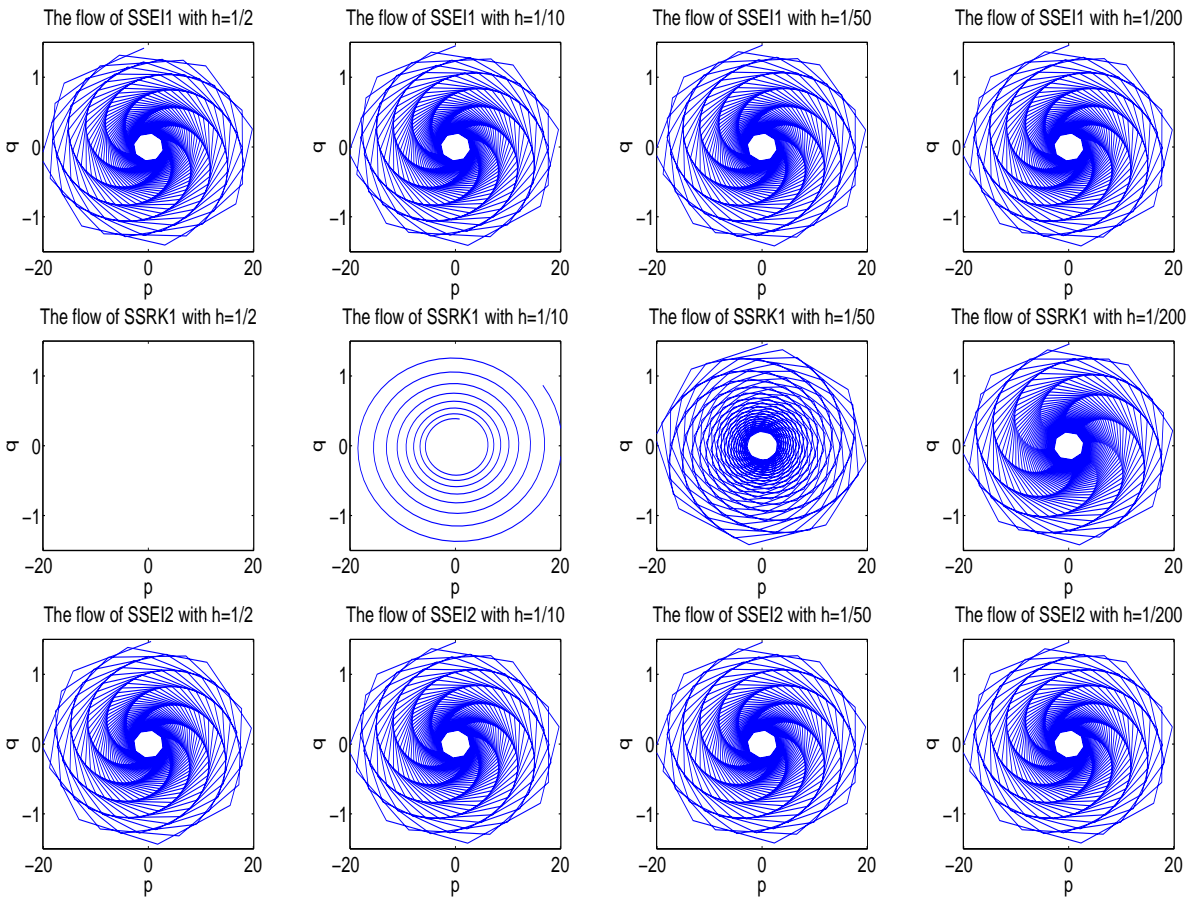

The flow of SSRK2 with $h=1 / 2$
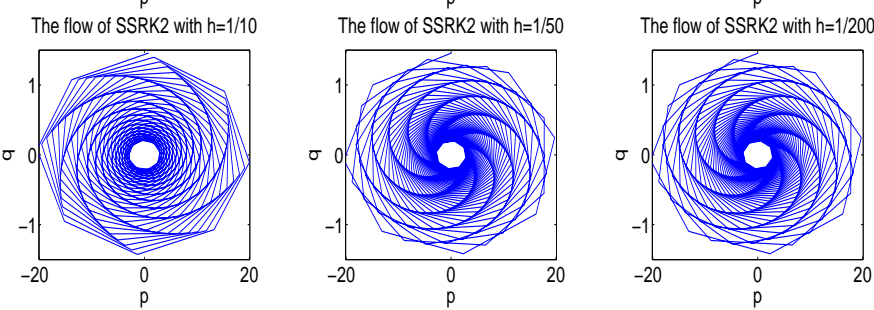

Figure 6: Problem 3: the flows of different methods.
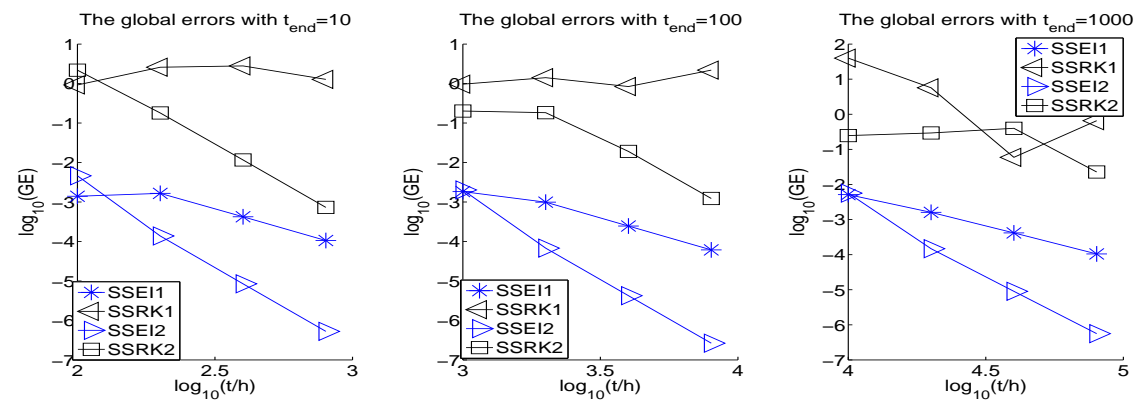

Figure 7: Problem 3: the relative global errors. 

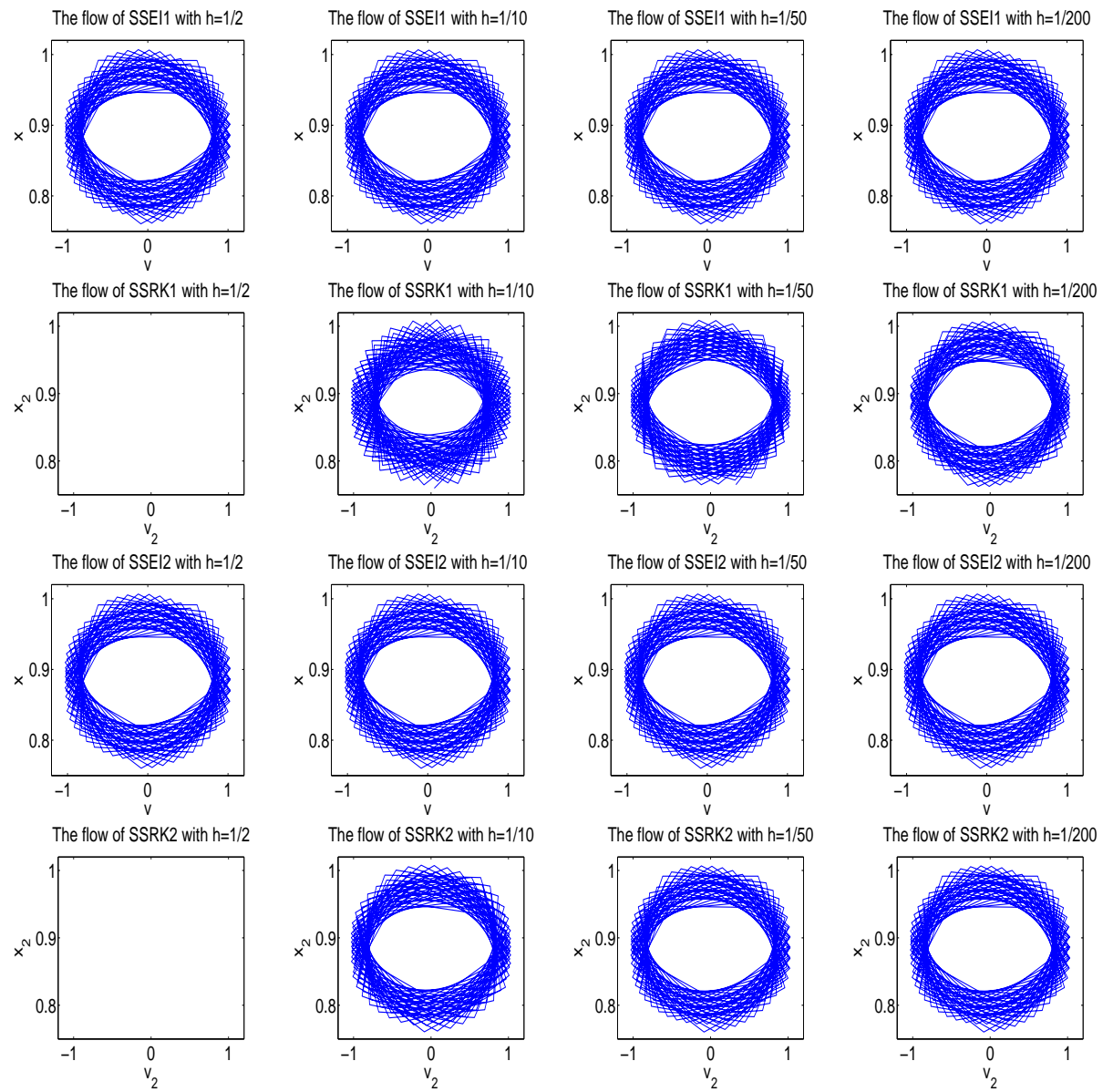

Figure 8: Problem 4: the flows of different methods.
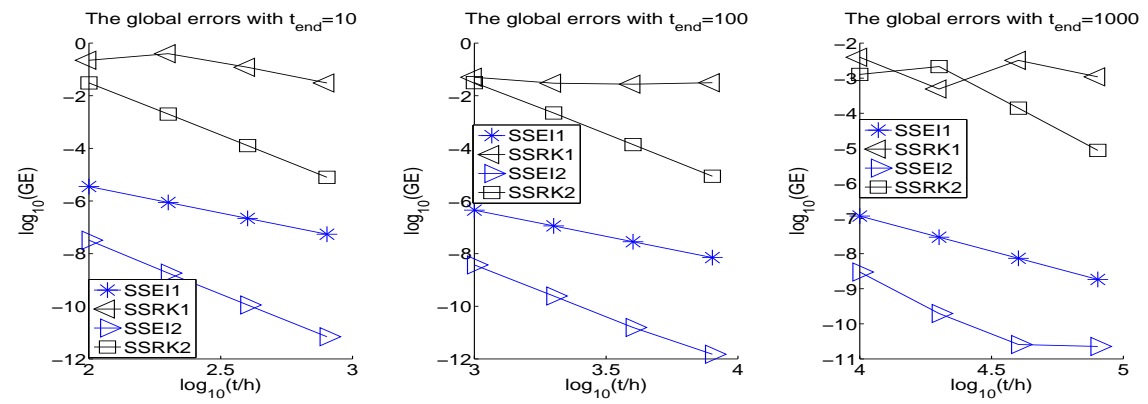

Figure 9: Problem 4: the relative global errors. 


\section{Conclusions}

This paper studied volume-preserving exponential integrators. The necessary and sufficient volumepreserving condition for exponential integrators was derived and volume-preserving properties were discussed for four kinds of vector fields. It was shown that symplectic exponential integrators can be of volume preservation for a much larger class of vector fields than Hamiltonian systems. It should be noted that a new result has been proved that all a kind of adapted exponential integrators methods is of volume preservation for the highly oscillatory system (22). Moreover, the volume-preserving property of ERKN/RKN methods is discussed for separable partitioned systems. Some new results on Geometric Integration were presented for second-order highly oscillatory problems and separable partitioned systems. We also carried out four numerical experiments to demonstrate the remarkable robustness and superiority of volume-preserving exponential integrators in comparison with volumepreserving Runge-Kutta methods.

\section{References}

[1] P. Badera, D. I. McLarena, G.R.W. Quispela, and M. Webb, Volume preservation by Runge-Kutta methods, Appl. Numer. Math., 109 (2016), pp. 123-137.

[2] L. Brugnano, G. Frasca Caccia, and F. Iavernaro, Hamiltonian Boundary Value Methods (HBVMs) and their efficient implementation, mathematics in engineering, science and aerospace mesa, 5 (2014), pp. 343-411.

[3] E. Celledoni, R.I. Mclachlan, D.I. Mclaren, B. Owren, G.R.W. Quispel, and W.M. Wright, Energy-preserving Runge-Kutta methods, M2AN Math. Model. Numer. Anal., 43 (2009), pp. 645-649.

[4] P. Chartier And A. Murua, Preserving first integrals and volume forms of additively split systems, IMA J. Numer. Anal., 27 (2007), pp. 381-405.

[5] D. Cohen And E. HaIRer, Linear energy-preserving integrators for Poisson systems, BIT Numer. Math., 51 (2011), pp. 91-101.

[6] A. ElíAs-ZÚÑIga, Analytical solution of the damped Helmholtz-Duffing equation, Appl. Math. Lett. 25, (2012), pp. 2349-2353.

[7] K. Feng And M. QIn, Symplectic Geometric algorithms for Hamiltonian systems, SpringerVerlag, Berlin, Heidelberg, 2010.

[8] K. Feng And Z.J. Shang, Volume-preserving algorithms for source-free dynamical systems, Numer. Math., 71 (1995), pp. 451-463.

[9] E. HAIRER, Energy-preserving variant of collocation methods, J. Numer. Anal. Ind. Appl. Math., 5 (2010), pp. 73-84.

[10] E. Hairer And C. Lubich, Long-time energy conservation of numerical methods for oscillatory differential equations, SIAM J. Numer. Anal., 38 (2000), pp. 414-441.

[11] E. HAIRER AND C. Lubich, Symmetric multistep methods for charged-particle dynamics, SMAI J. Comput. Math., 3 (2017), pp. 205-218. 
[12] E. Hairer AND C. LuBich, Long-term analysis of the Störmer-Verlet method for Hamiltonian systems with a solution-dependent high frequency, Numer. Math., 134 (2016), pp. 119-138.

[13] E. Hairer, C. Lubich, And G. WAnner, Geometric Numerical Integration: StructurePreserving Algorithms for Ordinary Differential Equations, 2nd edn. Springer-Verlag, Berlin, Heidelberg, 2006.

[14] Y. He, Y. Sun, J. Liu, AND H. QIn, Volume-preserving algorithms for charged particle dynamics, J. Comput. Phys., 281 (2015), pp. 135-147.

[15] M. Hochbruck And A. Ostermann, Explicit exponential Runge-Kutta methods for semilineal parabolic problems, SIAM J. Numer. Anal., 43 (2005), pp. 1069-1090.

[16] M. Hochbruck and A. Ostermann, Exponential integrators, Acta Numer., 19 (2010), pp. 209-286.

[17] M. Hochbruck, A. Ostermann, And J. Schweitzer, Exponential rosenbrock-type methods, SIAM J. Numer. Anal., 47 (2009), pp. 786-803.

[18] A. Iserles, G.R.W. Quispel, And P.S.P. Tse, B-series methods cannot be volumepreserving, BIT Numer. Math., 47 (2007), pp. 351-378.

[19] Y.W. Li AND X. WU, Exponential integrators preserving first integrals or Lyapunov functions for conservative or dissipative systems, SIAM J. Sci. Comput., 38 (2016), pp. 1876-1895.

[20] R.I. McLachlan, H.Z. Munthe-KaAs, G.R.W. Quispel, and A. Zanna, Explicit volumepreserving splitting methods for linear and quadratic divergence-free vector fields, Found. Comput. Math., 8 (2008), pp. 335-355.

[21] R.I. McLachlan and G. R. W. Quispel, Discrete gradient methods have an energy conservation law, Discrete Contin. Dyn. Syst., 34 (2014), pp. 1099-1104.

[22] R.I. McLachlan and C. Scovel, A survey of open problems in symplectic integration, Fields Inst. Commun., 10 (1998), pp. 151-180.

[23] L. Mei And X. Wu, Symplectic exponential Runge-Kutta methods for solving nonlinear Hamiltonian systems, J. Comput. Phys., 338 (2017), pp. 567-584.

[24] G.R.W. QuisPel, Volume-preserving integrators, Phys. Lett. A, 206 (1995), pp. 26-30.

[25] J.M. Sanz-Serna, Symplectic integrators for Hamiltonian problems: An overview, in Acta Numerica 1992, A. Iserles, ed., Cambridge University Press, Cambridge, UK, (1992), pp. 243286.

[26] B. WAng, A. Iserles, AND X. Wu, Arbitrary-order trigonometric Fourier collocation methods for multi-frequency oscillatory systems, Found. Comput. Math., 16 (2016), pp. 151-181.

[27] B. Wang And X. Wu, Functionally-fitted energy-preserving integrators for Poisson systems, J. Comput. Phys., 364 (2018), pp. 137-152.

[28] B. Wang, X. Wu, And F. Meng, Trigonometric collocation methods based on Lagrange basis polynomials for multi-frequency oscillatory second-order differential equations, J. Comput. Appl. Math., 313 (2017), pp. 185-201. 
[29] B. Wang, X. Wu, F. Meng, and Y. Fang, Exponential Fourier collocation methods for solving first-order differential equations, J. Comput. Math., 35 (2017), pp. 711-736.

[30] B. WAng, H. YAng, AND F. Meng, Sixth order symplectic and symmetric explicit ERKN schemes for solving multi-frequency oscillatory nonlinear Hamiltonian equations, Calcolo, 54 (2017), pp. 117-140.

[31] X. Wu And B. Wang, Recent Developments in Structure-Preserving Algorithms for Oscillatory Differential Equations, Springer Nature Singapore Pte Ltd, 2018.

[32] X. Wu, B. WANG, AND W. SHI, Efficient energy preserving integrators for oscillatory Hamiltonian systems, J. Comput. Phys., 235 (2013), pp. 587-605.

[33] X. Wu, X. You, And B. Wang, Structure-preserving algorithms for oscillatory differential equations, Springer-Verlag, Berlin, Heidelberg, 2013.

[34] X. Wu, X. You, W. Shi, and B. Wang, ERKN integrators for systems of oscillatory second-order differential equations, Comput. Phys. Comm., 181 (2010), pp. 1873-1887.

[35] H. XUE AND A. ZANNA, Explicit volume-preserving splitting methods for polynomial divergence-free vector fields, BIT Numer. Math., 53 (2013), pp. 265-281.

[36] A. Zanna, Explicit volume-preserving splitting methods for divergence-free ODEs by tensorproduct basis decompositions, IMA J. Numer. Anal. 35, (2014), pp. 89-106. 